CORRECTION

https://doi.org/10.1038/s41586-018-0690-1

\title{
Publisher Correction: Principles of nucleosome organization revealed by single-cell micrococcal nuclease sequencing
}

Binbin Lai, Weiwu Gao, Kairong Cui, Wanli Xie, Qingsong Tang, Wenfei Jin, Gangqing Hu, Bing Ni \& Keji Zhao

Correction to: Nature https://doi.org/10.1038/s41586-018-0567-3, published online 26 September 2018.

In Fig. 1c of this Letter, an error during the production process meant that the graph in the left panel was duplicated in the right panel. The right panel of Fig. 1c has been corrected online. 